\title{
História da evolução da qualidade hospitalar: dos padrões a acreditação*
}

\author{
Hospital quality evolution history: from patterns to accreditation \\ Historia de la evolucion de la calidad hospitalar: de los padrones a acreditacion
}

\author{
Liliane Bauer Feldman', Maria Alice Fortes Gatto², Isabel Cristina Kowal Olm Cunha ${ }^{3}$
}

\begin{abstract}
RESUMO: Trata-se da revisão da literatura sobre a padronização da Qualidade e o processo de avaliação pela Acreditação Hospitalar. Foi realizado um apanhado histórico sobre o início da avaliação da qualidade nos serviços de saúde, com o Programa de Padronização Hospitalar elaborado pelo Colégio Americano de Cirurgiões, em meados de 1924, até a criação da Organização Nacional de Acreditação - ONA, que tem como objetivos a implantação e implementação de um processo permanente de melhoria da assistência à saúde estimulando os serviços a atingirem padrões mais elevados de qualidade. Após reflexão, considerou-se que nas avaliações dos serviços faz-se necessário buscar a valorização dos resultados assistenciais bem como a elaboração de padrões quali - quantitativamente mais equânimes nos aspectos estrutura, processos e resultados da qualidade assistencial.
\end{abstract}

Descritores: Controle de qualidade; Acreditação; Administração hospitalar; Avaliação; Serviços de saúde

ABSTRACT: This work contemplates the literature revision of Quality Standardization and the evaluation process by means of Hospital Accreditation literature. A historic resume have been worked out, about the beginnings of quality evaluation within the health services, from the Hospital Standardization Program, elaborated by the American Surgeons College, in the middle of 1924, to the creation of ONA - National Accreditation Organization, who has as main goals the installation and implementation of a health assistance permanent improvement process, thus stimulating the services to reach higher quality standards. After a reflection exercise, it was considered that, in the evaluation of services, it's necessary to seek a higher value of the assistentials results, as well as the elaboration of more equanimous patterns in the structure, processes and results of the assistential quality.

Keywords: Quality control; Accreditation; Hospital administration; Evaluation; Health services

RESUMEN: Trata-se de una revisión de la literatura sobre padronización de Calidad y el procedimiento de evaluación por la Acreditación Hospitalar. Fue realizado un breve histórico sobre el inicio de la evaluación de calidad en los servicios de salud, con el Programa de Padronización Hospitalar creado por el Colegio Americano de Cirujanos, a medios de 1924, hasta la creación de la Organización Nacional de Acreditación - ONA, que tiene como objetivos la implementación de un proceso permanente de mejoría de la asistencia a la salud, haciendo que los servicios lleguen a los mejores niveles de cualidad. Después de un auto-análisis, se hizo necesaria que en las evaluaciones de servicios, se buscara la valorización de los resultados asistenciales bien como la elaboración de padrones cuali cuantitativamente más uniformes en aspectos de estructura, procesos e resultados de calidad asistencial.

Descriptores: Control de calidad; Acreditación; Administración hospitalaria; Evaluación; Servicios de salud

\section{INTRODUÇÃO}

A Avaliação da Qualidade na saúde iniciou-se no século passado, quando foi formado o Colégio Americano de Cirurgiões (CAC) que estabeleceu, em meados de 1924 o Programa de Padronização Hospitalar - $\mathrm{PPH}^{(1)}$. Neste programa foi definido um conjunto de padrões mais apropriados para garantir a qualidade da assistência aos paci- entes (Anexo1). Nele, três padrões relacionavam-se à organização do corpo médico, ao exercício da profissão, e ao conceito de corpo clínico; outro preconizava o preenchimento do prontuário, incluindo a história e exames do paciente, bem como as condições da alta; e o último, referia-se à existência de recursos diagnósticos e terapêuticos, além de no mínimo um laboratório clínico para análises e departamento de radiologia.

* Capitulo da Dissertação de Mestrado apresentada a Universidade Guarulhos em 2002.

1 Enfermeira. Mestra em Ciências da Saúde. Especialista em Administração Hospitalar. Proprietária-sócia da Consultoria Saúde Brasil Responsabilidade Profissional/ Hospitalar e Gerenciamento de Riscos. E-mail: lilibf@terra.com.br

2 Enfermeira. Doutora em Ciências da Saúde. Professora Aposentada do Depto de Enfermagem Médico Cirúrgico da USP. Professora do Curso de Pós-graduação da UNG.Orientadora.

3 Enfermeira. Doutora em Saúde Pública. Professora Adjunta do Depto Enfermagem da UNIFESP. Diretora da Faculdade de Enfermagem da UNISA. 
Estes padrões referiam-se às condições necessárias aos procedimentos médicos e ao processo de trabalho, não levando em consideração outras necessidades e ou serviços como o dimensionamento da equipe de enfermagem ou a necessidade da assistência 24 horas $^{(2)}$. Nem a avaliação dos resultados com o paciente e ainda poucos elementos da estrutura física do hospital.

Em 1918 foi realizada a primeira avaliação de hospitais nos Estados Unidos. De 692 hospitais com 100 leitos avaliados, apenas 89 cumpriram os padrões preconizados pelo PPH. Em contrapartida em 1950, o número de hospitais aprovados pela avaliação do PPH chegou a $3290^{(1-2)}$.

Em 1949, o Manual de Padronização mais desenvolvido, apresentava 118 páginas, mas o Colégio Americano de Cirurgiões passou a ter dificuldade em mantê-lo. Em parte devido à elevação dos custos, à sofisticação crescente da assistência médica, o aumento do número de instituições, a complexidade e a grande procura de especialidades não cirúrgicas após a $2^{\mathrm{a}}$.Guerra Mundial. A partir daí o CAC iniciou parcerias com Associação Médica Americana, Associação Médica Canadense, Colégio Americano de Clínicos e Associação Americana de Hospitais para apoio e participação com outras organizações inteiramente dedicadas à melhoria e promoção da acreditação voluntária ${ }^{(3)}$.

Com a união destes grupos nos Estados Unidos, em 1951, foi criada a Comissão Conjunta de Acreditação dos Hospitais (CCAH) que logo, em dezembro de 1952, delegou oficialmente o programa de Acreditação a Joint Commission on Accreditation of Hospitals. Esta era uma empresa de natureza privada, que na ocasião procurou introduzir e enfatizar na cultura médico-hospitalar a qualidade em nível nacional. Esta "cultura da qualidade" divulgou-se nos espaços acadêmicos e institucionais o que forçou a aprovação de leis mais complexas na área da saúde ${ }^{(4)}$, bem como passaram a ser enfatizados os aspectos de avaliação, educação e consultoria hospitalar.

$\mathrm{Na}$ década de 60 , como a maior parte dos hospitais americanos já havia atingido os padrões mínimos preconizados inicialmente, a Joint buscou então modificar o grau de exigência. Com isso, em 1970, publicou o Accreditation Manual for Hospital contendo padrões ótimos de qualidade, considerando também processos e resultados da assistência ${ }^{(2)}$.

Nos últimos anos, a Joint Commission on Accreditation of Healthcare Organization (JCAHO) passou a direcionar sua atuação no sentido de privilegiar a ênfase na assistência clínica através do monitoramento de indicadores de desempenho ajustados à gravidade, ao desempenho institucional e, finalmente assumiu recentemente o papel de educação com monitoramento, vistos na atividade de consultoria e na publicação de uma série de documentos como normas, padrões e recomendações ${ }^{(2)}$. A mensuração de resultados passou a ser expres- so em 4 níveis: acreditação com distinção, acreditação sem recomendação, acreditação com recomendação e acreditação condicional.

Como a saúde foi uma das últimas organizações sociais a adotar os modelos de qualidade, sua utilização iniciou-se timidamente na área administrativa. Um dos fatores que vem contribuindo para superar esta situação ${ }^{(5)}$ é a disputa de mercado entre as instituições hospitalares, pouco evidenciada em nosso meio, mas muito forte e acirrada em outros países. A partir do ano 2000, alterando o paradigma anterior, observa-se nos hospitais da região central de São Paulo mudança no padrão de atendimento e na prestação de serviços.Hoje se enfatiza a qualidade na assistência a saúde dentro de um mercado competitivo(6).

Assim, este artigo parte integrante de uma dissertação de mestrado tem como objetivo fazer uma revisão bibliográfica sobre os procedimentos de controle da qualidade assistencial entende-se aqui meios, métodos, estudos e ações de verificação e avaliação da assistência prestada na busca da qualidade, iniciados após a Segunda Guerra Mundial com o Programa de Padronização Hospitalar até a criação da Organização Nacional de Acreditação (ONA) no Brasil em maio de 1999.

Para consulta utilizou-se as fontes bibliográficas virtuais com as palavras chaves acreditação, avaliação dos serviços de saúde e qualidade hospitalar bem como pesquisou-se em bibliotecas artigos, revistas, publicações e estudos realizados até o ano de 2001. Foram encontrados 31 artigos dos quais 20 foram utilizados nesta pesquisa.

\section{Padronização e classificação dos hospitais}

O termo Qualidade ou Melhoria Contínua da Qualidade nos conceitos mais modernos é um fenômeno continuado de aprimoramento, que estabelece progressivamente os padrões, resultado dos estudos de séries históricas na mesma organização ou de comparação com outras organizações semelhantes, em busca do defeito zero - situação que, embora não atingível na prática, orienta e filtra toda ação e gestão da qualidade. É também um processo essencialmente cultural e desta forma envolve motivação, compromisso e educação dos participantes da entidade, que são assim estimulados a uma participação de longo prazo no desenvolvimento progressivo dos processos, padrões e dos produtos da entidade ${ }^{(7)}$.

Entende-se Qualidade

como um processo dinâmico, ininterrupto e de exaustiva atividade permanente de identificação de falhas nas rotinas e procedimentos, que devem ser periodicamente revisados, atualizados e difundidos, com participação da alta direção do hospital até seus funcionários mais básicos ${ }^{(4)}$. 
No Brasil, talvez o primeiro estudo no sentido de melhorar a qualidade na organização dos hospitais tenha sido de Odair Pedroso em 1935, ao conceber uma Ficha de
Inquérito Hospitalar (Quadro1) para a Comissão de Assistência Hospitalar do Ministério da Saúde, substituída posteriormente pelo Serviço de Medicina Social, hoje extinto ${ }^{(8)}$.

Quadro 1 - Ficha de Inquérito Hospitalar.São Paulo, 1935.

1.Corpo clínico organizado, com obrigatoriedade de médico plantonista residente

2.Corpo administrativo.

3.Corpo de Enfermeiros e auxiliares em número proporcional à capacidade e serviços clínicos do hospital, inclusive para plantão noturno.

4.Serviços radiológico e fisioterápico.

5.Laboratório clínico.

6.Necrotério com equipamento para necropsia.

7.Salas de operações com equipamentos suficientes e anexos.

8.Farmácia.

9.Serviços auxiliares (cozinha,lavanderia,desinfecção).

Fonte: Carvalho L. Padrões mínimos de organização de hospitais. Rev Paul Hosp. 1973; $21(3)$ : 107.

No sistema de saúde brasileiro, as iniciativas de classificação e categorização de hospitais e outros serviços de saúde sempre pertenceram ao poder público. Os esforços nesse sentido datam da década de 30, com o Censo Hospitalar do Estado de São Paulo, no qual formulou-se uma primeira proposta de regionalização e hierarquização de serviços, que não chegou a ser imple- mentada, mas serviu de inspiração para outras classificações criadas posteriormente ${ }^{(7)}$.

Em 1951, com o $1^{\circ}$.Congresso Nacional do Capítulo Brasileiro do Colégio Internacional de Cirurgiões realizado em São Paulo foram estabelecidos os primeiros padrões mínimos para Centro Cirúrgico e estudados tanto os aspectos de planta física como a organização desta unidade hospitalar, descritos no Quadro 2.

Quadro 2 - Padrões mínimos para Centro Cirúrgico do hospital.São Paulo, 1951.

1) Chefia: A administração do Centro cirúrgico será exercida por uma enfermeira, devidamente treinada.

2) Pessoal: Deverá ter número suficiente, devidamente treinado.

3) Regulamento: O regulamento do Centro cirúrgico deverá fazer parte do Regulamento do Hospital.

4) Rotinas: Deverá ser assegurado o seu bom funcionamento e divulgado a todos.

5) Avaliação do trabalho profissional: Será organizado um fichário contendo elementos que permitam avaliar a capacidade profissional do cirurgião.

6) Prontuário médico: a)nenhum doente poderá ser operado sem possuir prontuário médico que deverá conter o diagnóstico provisório; b)ao citado prontuário serão anexados: descrição do ato cirúrgico, ficha de anestesia, diagnóstico operatório e evolução pós-operatória, devendo o prontuário ser assinado pelo médico responsável.

Fonte: Carvalho L. Padrões mínimos de organização de hospitais. Rev Paul Hosp 1973; 21(3):107.

Neste Congresso, foram ainda estabelecidos os componentes do prontuário médico, bem como algumas normas gerais para a organização do hospital, indispensáveis ao bom funcionamento do setor ${ }^{(8)}$.

No âmbito federal, o Decreto 25465 de 1956 propõe Padrões Gerais, segundo as quais os distintos estabelecimentos de saúde podem ser classificados ${ }^{(7)}$.

Em 1960, o Instituto de Aposentadoria e Pensões dos Previdenciários já possuía padrões para credenciamento dos serviços hospitalares. Tais critérios abrangiam: planta física (23 itens - 300 pontos), equipamento (10 itens - 220 pontos) e organização (10 itens 480 pontos), especificando itens para a área "estrutura" como é classificada ainda hoje. O Instituto estabeleceu também o Relatório de Classificação Hospitalar Reclar, que possuía três áreas: planta física, equipamento e organização, num total de 333 itens. Este relatório, porém, sofreu crítica devido à excessiva ênfase aos aspectos físicos e a pouca importância aos recur- sos humanos, e quase nenhuma menção à produção e produtividade (processo), sem falar em resultados ${ }^{(9)}$. Atualmente estes critérios continuam válidos e foram apenas acrescidos outros descritos em manuais e livros, complementando o processo inicial.

\section{Avaliação da Qualidade pelo Processo de Acreditação Hospitalar}

Acreditação é uma palavra originária do inglês, utilizada pelo Manual Brasileiro de Acreditação e pelo Manual das Organizações Prestadoras de Serviços Hospitalares ${ }^{(10-11)}$.

A Acreditação é o procedimento de avaliação dos recursos institucionais, voluntário, periódico, reservado e sigiloso, que tende a garantir a qualidade da assistência através de padrões previamente aceitos.Os padrões podem ser mínimos (definindo o piso ou base) ou mais elaborados e exigentes, definindo diferentes níveis de satisfação e qualificação como complementam ${ }^{(4)}$. 
Na tentativa de identificar os primórdios da idéia de acreditação, buscou-se o estudo recente ${ }^{(2)}$ que resgata a evolução histórica da acreditação hospitalar. Nele, é citado que, originalmente os sistemas de acreditação foram desenhados para proteger e organizar a profissão médica, aprimorando o ambiente e a prática clínica. Com o passar do tempo o trabalho estendeu-se para outras áreas de assistência à saúde com outras finalidades como educação, consultoria, participação e profissionalismo.

Desde 1970, o Ministério da Saúde desenvolve o tema Qualidade e Avaliação Hospitalar partindo de início da publicação de Normas e Portarias a fim de regulamentar esta atividade e atualmente trabalha na implantação de um sistema eficaz e capaz de controlar a assistência à saúde no Brasil ${ }^{(3)}$.

Para a Organização Mundial da Saúde - OMS a partir de 1989, a Acreditação passou a ser elemento estratégico para o desenvolvimento da qualidade na América Latina. Em 1990 foi realizado um convênio com a Organização Pan-Americana de Saúde - OPAS, a Federação Latino Americana de Hospitais e o Ministério da Saúde para elaborar o Manual de Padrões de Acreditação para América Latina ${ }^{(2,4)}$.

Quatro grupos de trabalho formaram-se em São Paulo, Rio Grande do Sul, Paraná e Rio de Janeiro, pois a intenção do Ministério da Saúde em associar-se a diferentes grupos foi consolidar diferentes experiências em uma metodologia única e de consenso para começar a implementar o Programa em nível nacional ${ }^{(12)}$.

Das reuniões de discussão deste tema em São Paulo, participaram a Associação Paulista de Medicina - APM, o Conselho Regional de Medicina do Estado de São Paulo - CREMESP, o Sindicato dos Hospitais do Estado de São Paulo - SINDHOSP, o Conselho Regional de Enfermagem do Estado de São Paulo - COREN, entre outros. Destes, permaneceram apenas a APM e o CREMESP, dando origem ao Programa de Controle de Qualidade do Atendimento Médico-Hospitalar - CQH no Estado de São Paulo, pioneiro no Brasil ${ }^{(13)}$.

No Rio Grande do Sul, em 1995, o Instituto de Administração Hospitalar e Ciências da Saúde, em associação com a Secretaria Estadual da Saúde e do Meio Ambiente e o SEBRAE/RS, desenvolveu um projeto de pesquisa com o propósito de determinar padrões de qualidade hospitalar ${ }^{(14)}$.

Também em 1995, a Secretaria de Estado da Saúde do Paraná, baseada na experiência com o Programa de Controle de Infecção Hospitalar, estendeu a preocupação com a qualidade das organizações, utilizando para tanto o processo de acreditação ${ }^{(2)}$.

No Rio de Janeiro, em agosto/setembro de 1997, o Projeto de Acreditação e Certificação da Qualidade em Saúde - PACQS transformou-se no Consórcio Brasileiro de Acreditação - CBA, com a Fundação Cesgranrio, criada pelas Universidades Estaduais do Rio para avaliação do processo educacional. A Fundação implantou a avaliação também na área da saúde ${ }^{(2)}$, trazendo para o cenário da acreditação outra faceta da avaliação: a de ramo de negócios.

Em dezembro de 1997, o CBA realizou um seminário com a assessoria da Joint Commission para elaborar um programa nacional de acreditação de hospitais. Nesta ocasião, com esta associação junto a Joint Commission, o Brasil foi integrado ao contexto internacional de avaliação de serviços de saúde ${ }^{(2)}$.

Retomando o desenvolvimento da Acreditação na América Latina, em abril de 1992, a OPAS promoveu em Brasília, o primeiro Seminário Nacional sobre Acreditação, no qual foi apresentado o Manual de Acreditação da OPAS. Neste seminário surgiram algumas conclusões sobre como controlar a qualidade dos serviços de saúde, destacando-se que a acreditação seria um mecanismo válido para controlar a qualidade. O Manual de Acreditação proposto seria um instrumento básico importante para iniciar o processo e os participantes levariam a discussão para suas entidades ${ }^{(3)}$.

Em 1994, o Ministério da Saúde lançou o Programa de Qualidade com o objetivo de promover esta cultura. Estabeleceu ainda a Comissão Nacional de Qualidade e Produtividade em Saúde - CNQPS que desempenhou importante papel na elaboração das diretrizes do programa e na sua disseminação, inclusive em outras esferas do governo. Ainda em março de 1994, o Instituto de Medicina Social da Universidade Estadual do Rio de Janeiro, a Academia Nacional de Medicina e o Colégio Brasileiro de Cirurgiões organizaram o Simpósio "Acreditação de Hospitais e Melhoria de Qualidade em Saúde" do qual participaram, entre outros os representantes da Joint Commission e do Canadian Council on Health Services Accreditation e entidades nacionais.

O Programa Brasileiro de Qualidade e Produtividade - PBQP estabeleceu a Avaliação e Certificação de Serviços de Saúde como sendo um projeto estratégico prioritário do Ministério da Saúde, para o biênio 97/98. Em julho de 1997, o Ministério da Saúde anunciou medidas para desenvolver a Acreditação na tentativa de unificar os esforços nacionais. Com isto iniciou o projeto junto ao REFORSUS com o Programa de Apoio Financeiro para o Fortalecimento do Sistema Único de Saúde, e o financiamento pelo Banco Mundial, chamado Acreditação Hospitalar ${ }^{(12,15)}$.

O Programa Brasileiro de Acreditação foi oficialmente lançado em novembro de 1998, no Congresso Internacional de Qualidade na Assistência a Saúde em Budapeste, bem como o instrumento nacional desenvolvido e conduzido por Humberto de Moraes Novaes.

Posteriormente, foram elaboradas propostas para o Sistema Nacional de Acreditação, ou seja, as normas 
básicas do processo de acreditação: credenciamento de instituições acreditadoras, qualificação e capacitação de avaliadores, código de ética e programa brasileiro de acreditação hospitalar ${ }^{(11)}$.

\section{Organização Nacional de Acreditação - ONA}

No período entre 1998 e 1999, o Ministério da Saúde realizou o projeto de divulgação da "Acreditação no Brasil". Constituiu-se de um ciclo de palestras envolvendo 30 localidades, em âmbito nacional, entre elas as 27 capitais de Estado, atingindo desta forma, todas as regiões do país.

O ciclo de palestras teve como objetivo apresentar o projeto desenvolvido pelo Ministério, para sensibilizar e melhorar a compreensão sobre o Sistema Brasileiro de Acreditação bem como sua forma de operacionalização o que culminou com a criação da entidade ONA - Organização Nacional de Acredi-tação, em maio de 1999.

As palestras atingiram um público de aproximadamente 6000 pessoas, entre gestores de serviços de saúde, profissionais da área, organizações de classe e empresas ligadas ao setor ${ }^{(11,15)}$.

A divulgação do Processo de Acreditação teve um resultado positivo se forem considerados os esclarecimentos levados aos representantes dos serviços de saúde que até o momento poucas informações tinham. Podemos considerar que este momento foi delineado pelo plantio da semente do "Processo de Acreditação" no Brasil.

A ONA é uma organização privada, sem fins lucrativos e de interesse coletivo, que tem como principais objetivos à implantação e implementação nacional de um processo permanente de melhoria da qualidade da assistência à saúde, estimulando todos os serviços de saúde a atingirem padrões mais elevados de qualidade, dentro do Processo de Acreditação ${ }^{(16)}$.

As Instituições Acreditadoras são empresas de direito privado, credenciadas pela ONA, que tem a responsabilidade de proceder à avaliação e à certificação da qualidade dos serviços de saúde em âmbito nacional.

A organização prestadora de serviços de saúde que aderir ao processo de acreditação estará revelando a sua res-ponsabilidade e o seu comprometimento com segurança, com a ética profissional, com procedimentos que realiza e com a garantia da qualidade do atendimento à população ${ }^{(16)}$.

O objetivo dos padrões da Acreditação é ficar à frente dos últimos progressos técnicos nas organizações hospitalares. Se permitirmos que os padrões sejam mínimos, não teremos progressos técnicos ${ }^{(17)}$.

O processo avaliatório da Acreditação dá-se a partir de padrões previamente estabelecidos, isto é, comparase o que é encontrado nos serviços com o padrão considerado como referência. Assim sendo, verifica-se se o serviço atinge ou não os critérios preconizados como desejáveis $^{(18)}$.

Itens de verificação apontam as fontes onde os avaliadores podem procurar as provas, ou o que o hospital puder apresentar para indicar que cumpre com determinado padrão e em que nível. Estas fontes podem ser documentos do hospital, entrevistas com as chefias de serviço, funcionários, clientes e familiares, prontuários médicos, registros dos pacientes e outros ${ }^{(10)}$.

A fase de avaliação e visita é composta por dois grandes momentos: a pré-visita e a visita propriamente dita. Na pré-visita, o hospital prepara-se para o processo com a divulgação interna e distribuição do Manual aos funcionários. A visita somente ocorrerá após a solicitação formal voluntária do hospital, à Instituição Acreditadora.

A duração da visita dos avaliadores é variável em função do porte e da complexidade do hospital. Na visita, todos os setores e unidades são avaliados dentro de uma programação definida, junto aos responsáveis do hospital $^{(10)}$.

O relatório é elaborado pelos avaliadores para o Hospital e para a gerência da Instituição Acreditadora com um parecer final dos avaliadores (de consenso) sobre a indicação para a acreditação e em que nível. O Certificado é emitido pela Instituição Acreditadora e tem a validade de dois anos, para "Acreditado" e "Acreditado pleno". Já o "Acreditado com Excelência" tem prazo de validade de três $\operatorname{anos}^{(19)}$. Terminados estes prazos, a instituição deve submeter-se a um novo processo de avaliação.

Em estudo recente sobre Critérios de Avaliação do Serviço de Enfermagem que são utilizados nos Processos de Acreditação Institucional, identificou-se que no âmbito nacional foram avaliados 195 hospitais no período de outubro de 1999 a março de 2002. No Brasil, de um universo de 6.528 instituições de saúde, 36 foram Acreditadas do total de 195 avaliadas. Destas, apenas 27 instituições conseguiram manter o nível de conformidade e conseqüentemente o Certificado de Hospital Acreditado. Em processo de acreditação estavam 79 instituições neste período ${ }^{(20)}$.

Em relação aos critérios de avaliação dos serviços de enfermagem identificados no estudo, há ênfase predominante nos critérios de estrutura e processos organizacionais sobre os critérios de resultados institucionais. Portanto, verificou-se que nas avaliações dos serviços faz-se necessário mudar os mecanismos tradicionais de avaliação, centrados na estrutura física, no faturamento de serviços produzidos e na hotelaria; para buscar-se a valorização dos resultados assistenciais advindos de programações com critérios epidemiológicos, padrões e indicadores de qualidade, competência e desempenho com excelência, elaboração de padrões quali - quantitativamente mais equânimes nos aspectos estrutura, processos e resultados. 
Considerando as avaliações externas como meio de implementar a equidade na elaboração de critérios, existem cinco iniciativas atualmente no Brasil: ISO, Prêmio Nacional de Qualidade, Acreditação, Auditoria Médica e Análise de Riscos Profissionais Legais ${ }^{(19,21)}$.

Ressalta-se no contexto das avaliações da qualidade, o papel integra-dor da ONA, que desenvolve um trabalho minucioso de unificação das diversas iniciativas de avaliação fortalecendo o sistema de saúde no Brasil ${ }^{(19)}$.

\section{CONSIDERAÇÕES FINAIS}

A certificação da avaliação hospitalar pelo processo de Acreditação aponta uma direção positiva na melhoria da assistência aos pacientes, bem como estabelece níveis crescentes de Qualidade.Os profissionais da área de enfermagem têm contribuído para o desenvolvimento da qualidade assistencial e institu-cional participando dos processos avaliativos em situações distintas, isto é, em determinadas condições o profissional e seu trabalho são avaliados e em outros momentos, o enfermeiro é um dos agentes avaliadores como nos casos da acreditação e da avaliação dos riscos profissionais-legais.

A padronização dos processos de avaliação vem ao longo dos anos evoluindo e aprimorando a identificação de critérios, indicadores e padrões cada vez mais significativos para os vários serviços hospitalares.

Entende-se que o futuro será das organizações que possuírem algum tipo de certificação e que de certa forma, isto quebrará o paradigma da fragmentação da saúde proporcionando serviços que realmente atendam as necessidades da população, com segurança, inclusive superando as expectativas dos pacientes, essência da excelência.

\section{REFERÊNCIAS}

1. Roberts JC, Coale JG, Redman MA. A history of the joint commission of accreditation of hospitals. JAMA 1987; 258(7):936-40.

2. Schiesari LM. Cenário da acreditação hospitalar no Brasil: evolução histórica e referências externas.[dissertação mestrado]. São Paulo: Faculdade de Saúde Pública da USP; 1999.

3. Anais do Simpósio Acreditação de Hospitais e Melhoria da Qualidade em Saúde; 1994, Rio de Janeiro (RJ). An Acad Nac Med 1994; 154(4):185-213.

4. Novaes HM, Paganini JM. Desenvolvimento e fortalecimento dos sistemas locais de saúde na transformação dos sistemas nacionais de saúde: padrões e indicadores de qualidade para hospitais (Brasil). Washington (DC): Organização Panamericana de Saúde; 1994. (OPAS/HSS/94.05).
5. Antunes AV. O gerenciamento da qualidade na enfermagem. [tese doutorado]. Ribeirão Preto: Escola de Enfermagem de Ribeirão Preto/USP; 1997

6. Feldman LB. Como alcançar a qualidade nas instituições de saúde: critérios de avaliações, procedimentos de controle, gerenciamento de riscos hospitalares até a certificação. São Paulo: Martinari; 2004.

7. Azevedo AC. Indicadores de Qualidade e Produtividade em Serviços de Saúde. Rev Ind Qual Produt Ipea 1993; 1(1):49-54.

8. Carvalho LF. Padrões mínimos de organização de hospitais. Rev Paul Hosp 1973; 21(3):107.

9. Azevedo AC. Contribuição à metodo-logia de análise de hospitais complexos. [tese livre-docência]. São Paulo: Faculdade de Saúde Pública da USP; 1989.

10. Ministério da Saúde. Secretaria de Políticas da Saúde. Departamento de Avaliação de Políticas de Saúde. Manual brasileiro de acreditação hospitalar. $2^{\mathrm{a}}$ ed. Brasília (DF); 1999.

11. Ministério da Saúde. Organização Nacional de Acreditação. Manual das organizações prestadoras de serviços hospitalares. Brasília (DF); 2001.

12. Baptista BSF. El desarrollo del proceso de acreditación de hospitales en Brasil. Rev Itaes 2000; 1(5):15- 8.

13. Associação Paulista de Medicina (APM). Controle da qualidade do atendimento médico-hospitalar no Estado de São Paulo: manual de orientação aos hospitais participantes. $2^{\mathrm{a}}$ ed. São Paulo: Atheneu; 1998.

14. Quinto Neto A, Gastal FL. Acreditação hospitalar: proteção dos usuários, dos profissionais e das instituições de saúde. Porto Alegre: Dacasa; 1997.

15. Organização Nacional de Acreditação. Fundadoras Associadas Acreditadoras. [monografia online]. Disponível em: <http:// ona.terra.com.br/conteudoona/historico.html>. (08 maio 2001).

16. Organização Nacional de Acreditação. A saúde no Brasil: agora tem um processo permanente de avaliação e certificação da qualidade. Brasília (DF); 2000. [Folder].

17. Burmester H. Reflexiones sobre los programas hospitalarios de garantia de calidad. Rev Panam Salud Publica. 1997; 2(1): 149-54.

18. Malik AM, Schiesari LMC. Qualidade na gestão local de serviços e ações de saúde. São Paulo: Instituto para o Desenvolvimento da Saúde/ Universidade de São Paulo/Faculdade de Saúde Pública/ Banco Itaú; 1998. (Saúde Cidadania, 3).

19. Quinto Neto A, Bittar OJNV. Hospitais: administração da qualidade e acreditação de organizações complexas. Porto Alegre: Dacasa; 2004. 
20. Feldman LB. Analise dos critérios de avaliação dos serviços de enfermagem adotados nos processos de acreditação institucional. [dissertação mestrado]. Guarulhos (SP): Universidade de Guarulhos; 2002.
21. Feldman LB, Bordin TA, Mariona FG. Responsabilidade civil profissional - instituições de saúde: perfil do enfermeiro avaliador institucional. In: Anais do $53^{\circ}$. Congresso Brasileiro de Enfermagem; 2001; Curitiba (PR). Curitiba: ABEn - Seção PR; 2001.

\title{
Anexo 1
}

Quadro 3 - Os padrões mínimos preconizados pelo Colégio Americano de Cirurgiões, 1924.

\begin{abstract}
1.Médicos e cirurgiões com o privilégio de exercer a prática profissional no hospital devem estar organizados como um grupo ou um corpo clínico.

2. A admissão dentro do corpo clínico é restrita a médicos e cirurgiões que sejam graduados em Medicina com licença legal para a pratica em seus respectivos Estados ou províncias, competentes e valorosos em caráter e em relação à ética.

3. O corpo clínico inicia suas atividades com a aprovação do conselho diretor do hospital, adota regras, regulamentos e diretrizes no trabalho no hospital: a- reuniões do corpo médico ao menos mensalmente (em grandes hospitais podem optar por se reunir separadamente); b-revisão e análise da experiência clínica deve ser feita em intervalos regulares nos vários departamentos e o prontuário dos pacientes, deverá ser a base desta revisão e análise.

4. Os registros dos pacientes devem ser precisos e completos e devem estar escritos de forma acessível a todo hospital - inclui dados de identificação, queixa, história pessoal e familiar, história da moléstia atual, exame físico, exames especiais como consultas ou laboratório clínico ou raio-x, entre outros, hipótese diagnóstica, tratamento médico ou cirúrgico, achados patológicos, evolução clínica, diagnóstico final, condição de alta, seguimento, e, no caso de morte, achados de autópsia.

5. Recursos diagnósticos e terapêuticos, devem estar disponíveis para o estudo diagnóstico e tratamento dos pacientes, incluindo ao menos um laboratório clínico com serviços de análises químicas, bacteriologia, sorologia e patologia e departamento de raio x com serviços de radiografia e fluoroscopia.
\end{abstract}

Fonte: Roberts,JC; Coale,JG; Redman,MA. A history of joint commission on accreditation of hospitals. Jama. 1987; 258 (7): $936-40$. 